\title{
UNA STIMA DEL PUNTO DI FUSIONE DEL FERRO SOTTO ALTE PRESSIONI
}

\author{
P. E. VAlle
}

Introduzione - Le recenti teorie sulla costituzione del nucleo della Terra, sviluppate da W. Kuhn e A. Rittmann e da W. H. Ramsey (1), non sembrano indurre ad abhandonare l'ipotesi classica, secondo la quale il nucleo della Terra sarebhe composto da un materiale assai simile al ferro metcorico.

Uno dei metodi che possono servire a sostenere o meno l'ipotesi classica, consiste nel confronto della curva densità-pressione relativa al nucleo della Terra, con un'analoga curva calcolata per il ferro.

Esistono diverse curve densiti-pressione relative all'interno della Terra, ma le più recenti e attendibili, dovute a K. E. Bullen $\left({ }^{2-3}\right)$, non sono molto diverse fra loro.

Il calcolo della curva densiti-pressione per il ferro è stato eseguito da $\mathrm{H}$. Jensen $\left({ }^{t}\right)$, utilizzando il metodo Thomas-Fermi, e si riferisce allo zero assoluto della temperatura. Tale curva non è corretta nell'intervallo delle medie e hasse pressioni. Essa fornisce, a pressione ordinaria, un valore della densita inferiore al valore sperimentale. Analoghe curve, per vari elementi, sono state costruite da W. M. Elsasser (ji) in base ai risultati dei calcoli esegruiti da R. P. Feynman, N. Metropolis e E. Teller ("), sequendo la linea del metodo ThomasFermi.

Esiste inoltre un'isoterma del ferro, a temperatura ambiente, calcolata da F. Birch $1^{7}$ mediante la teoria delle deformazioni finite e i dati sperimentali di P. W. Bridgmann $\left({ }^{8}\right.$.

Per le ricerche sulla costituzione e sullo stato fisico del nucleo della Terra $\dot{e}$ anche di grande interesse la conoscenza dell'andamento della temperatura di fusione del ferro in funzione della pressione e la conoscenza della temperatura raggiungilile per compressione adiabatica.

Una stima recente della temperatura di fusione del ferro, in funzione della pressione, è stata effettuata da F. E. Simon (") per mezzo di una relazione semiempirica da lui stesso stabilita. 
In questa nota vengono esposti i risultati del calcolo della densità e temperatura del ferro al punto di fusione, in funzione della pressione, e i risultati del calcolo della temperatura ragrgiungilile per compressione adialoatica, a partire dalla temperatura di fusione a pressione nulla.

I calcoli sono stati eseguiti sulla base della teoria della fusione di Lindmann $\left({ }^{10}\right)$ e dell'equazione di stato dei solidi stabilita da P. E. Valle $\left({ }^{11}\right.$ \%.

Vengono inoltre effettuati confronti con i risultati di H. Jensen, F. Birch, F. E. Simon e con la curva densita pressione, relativa al nucleo della Terra, calcolata da K. E. Bullen.

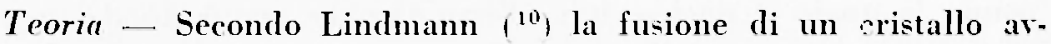
viene quando l'ampiezza delle oscillazioni degli atomi diventa ablyastanza grande da far entrare in collisione le "sfere d'azione " degli atomi stessi. Con l'ipotesi che la temperatura di fusione sia sufficientemente elevata, in modo che siano applicalsili le legrgi della dinamica classica, si perviene alla formula di Lindmann

$$
T_{s}=C A V^{2 / 3} v^{2}
$$

nella quale $T_{s}$ è la temperatura di fusione, $V_{s}$ il volume atomico. $A$ il peso atomico, v la frequenza di oscillazione e $C$ una costante.

Se si considera che nella fusione si ha una brusca diminuzione della rigidità, si è indotti ad assumere come frequenza la frequenza delle onde trasversali di agritazione termica, la quale ì data (11) da

$$
\nu_{1}=\nu_{0 t} e^{\gamma_{0 t}}\left(1-\frac{0_{0}}{0}\right)
$$

La formula di Lindmann si può quindi porre nella forma

$$
\frac{T_{s}}{T_{o s}}=\left(\frac{\rho_{o s}}{\rho_{s}}\right)^{\frac{2}{3}} e^{2 \gamma_{o t s}\left(1-\frac{\rho_{0 s}}{\rho_{s}}\right)}
$$

Questa relazione consente di calcolare la temperatura di fusione in funzione della densità della fase solida $\rho_{s}$ al punto di fusione. quando sia noto il parametro $\gamma_{\text {ots }}$ valutato alla densiti $p_{0 .}$.

É interessante notare che la temperatura di fusione presenta un massimo per $\rho_{s}=3 \gamma_{\text {ots }} \rho_{o s}$. Ciò significa che il passagrgio dalla fase solida alla fase liquida può avvenire a qualsiasi temperatura purché la pressione sia tale da rendere sufficientemente piccola la distanza media tra gli atomi. 
Peraltro la pressione relativa al massimo della temperatura di fusione è generalmente elevatissima e fuori dell'intervallo delle pressioni che si considerano in questa ricerca.

La pressione al punto di fusione può essere calcolata mediante l'equazione di stato. Se $p_{0}$ è la densità a pressione nulla e alla temperatura $T_{m}$, l'equazione di stato dei solidi, per alte temperature, si scrive (11)

$$
p=K_{\mathrm{o} \mathbf{T}} e^{2 \Gamma^{\top}}\left(2 \Gamma^{\prime}\right)^{1 / 3}\left[F\left(\frac{1}{2 \Gamma} \frac{\rho}{\rho_{\mathbf{0}}}\right)-F\left(\frac{1}{2 \Gamma}\right)\right]+\alpha_{\mathrm{o}} K_{\mathbf{o}_{\mathbf{T}}}\left(T-T_{\mathbf{o}}\right)
$$

Detta quindi $p_{s}$ la pressione al punto di fusione, pos la densità della fase solida al punto di fusione e a pressione nulla, $T_{\text {os }}$ la temperatura di fusione a pressione nulla, si arrà

$$
p_{s}=p_{1}+p_{2}
$$

dove

$$
p_{1}=K_{\mathrm{oT}} c^{2 \Gamma}(2 \Gamma)^{1 / 3}\left[F\left(\frac{\pi}{2 \Gamma} \frac{\rho_{s}}{\rho_{0}}\right)-F\left(\frac{1}{2 \Gamma} \frac{\rho_{o t}}{\rho_{0}}\right)\right]
$$

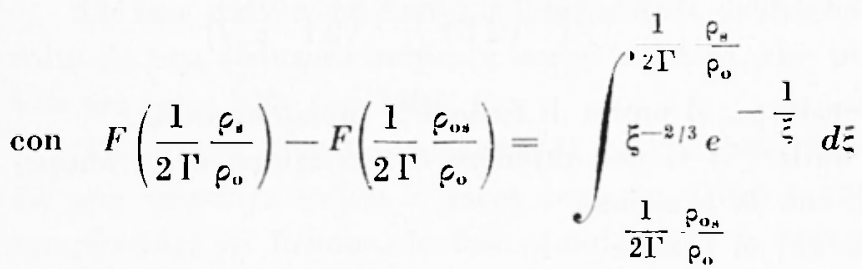

e

$$
p_{\mathrm{a}}=\alpha_{\mathrm{o}} K_{\mathrm{oT}}\left(T_{\mathrm{s}}-T_{\mathrm{od}}\right)
$$

La pressione $p_{1}$ è dovuta allinterazione fra le particelle del solido, mentre la pressione, " ¿ dovuta allagitazione termica e contribuisce con una percentuale piuttosto piccola alla pressione totale.

Indicando con $\gamma_{0}$ e $\hat{\gamma}_{\circ}$ i parametri delle onde di agitazione termica longitudinali e trasversali, con $v_{a 1}$ e $v_{o t}$ le velocità di tali onde relative alla densità $c_{0}\left({ }^{11}\right)$ si ha

$$
\begin{gathered}
\Gamma=\gamma_{01}+\frac{a^{2}}{1-a^{2}}\left(\gamma_{0 l}-\gamma_{o t}\right) \\
a^{2}=\frac{4}{3} \frac{v^{2}{ }_{\mathrm{ot}}}{v^{2}{ }_{\mathrm{ol}}}
\end{gathered}
$$


E opportuno poi ricordare che tra l'incompressibilità isoterma $K_{\mathrm{T}}$, la dilatazione termica $x$, il parametro di Guineisen $\gamma$ e il calore specifico a volume costante $C_{r}$, sussiste la relazione

$$
\alpha K_{\mathrm{r}}=\gamma_{\mathrm{C}} C_{\mathrm{v}}
$$

nella quale (11)

$$
\gamma=\frac{\gamma_{1}+2 \gamma_{t}}{3}
$$

e risulta

$$
\begin{aligned}
& \gamma_{1}=\text { cost. } \\
& \gamma_{c}=\text { cost } .
\end{aligned}
$$

mentre l'incompressibilità isoterma è data (11) da

$$
K_{\mathrm{T}}=K_{\mathrm{o}_{\mathrm{T}}}\left(\frac{\rho}{\rho_{\mathrm{o}}}\right)^{1 / 3} e^{2 \Gamma\left(1-\frac{\rho_{\mathrm{o}}}{\rho}\right)}
$$

L'equazione di stato [4] consente il calcolo di pos quando siano note tutte le altre grandezze. Posto infatti nella [4] $p=p_{o s}, p=0$, si ha

$$
\alpha_{\mathrm{o}}\left(T_{\mathrm{og}}-T_{\mathrm{o}}\right)=e^{2 \Gamma}(2 \Gamma)^{1 / 3}\left[F\left(\frac{1}{2 \Gamma}\right)-F\left(\frac{1}{2 \Gamma} \frac{\rho_{\mathrm{ot}}}{\rho_{\mathrm{o}}}\right)\right]
$$

che fornisce la densità pos al punto di fusione a pressione nulla.

Dalla [3] e dalla [5] si può ottenere la variazione della temperatura di fusione con la pressione.

Derivando la [3] si ottiene

$$
\frac{d T_{s}}{d P_{s}}=2-\frac{T_{y}}{\rho_{s}}\left(\rho_{0} \cdot \frac{\rho_{0 s}}{\rho_{s}}-\frac{1}{3}\right)
$$

Analogamente dalla [5] si ha

$$
\frac{d p_{\mathrm{s}}}{d \rho_{\mathrm{y}}}=\frac{K_{\mathrm{r}}}{\rho_{\mathrm{t}}}+\alpha_{\mathrm{o}} K_{\mathrm{o}_{\mathrm{T}}} \frac{d T_{\mathrm{s}}}{d \rho_{\mathrm{s}}}
$$

e quindi, combinando le due espressioni, risulta

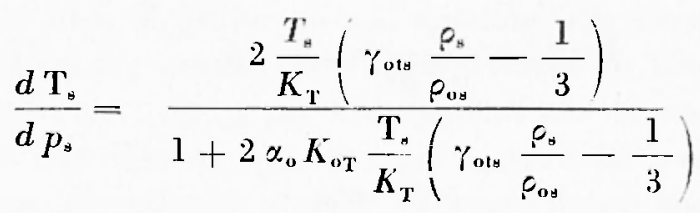

nella quale $K_{\mathrm{T}}$ è l'incompressibilità isoterma relativa alla densitì $G_{\mathrm{u}}$. 
i: interessante valutare l'andamento della temperatura durante una compressione adiabatica, a partire dal punto di fusione a pressione nulla. Indicando con $T_{n}$ tale temperatura si ha (12-13)

$$
T_{\mathrm{n}}=T_{\mathrm{os}} e^{\gamma_{\mathrm{os}}\left(1-\frac{\rho}{\rho_{\mathrm{os}}}\right)}
$$

mentre la pressione assume la forma

$$
P_{\mathrm{u}}=K_{\mathrm{oT}} e^{2 \mathrm{I}^{\prime}}(2 \mathrm{\Gamma})^{1 / 3}\left[F\left(\frac{1}{2 \Gamma} \frac{\rho}{\rho_{\mathrm{c}}}\right)-F\left(\frac{1}{2 \mathrm{\Gamma}} \frac{\rho_{\mathrm{oH}}}{c_{\mathrm{o}}}\right)\right]+\alpha_{\mathrm{o}} K_{\mathrm{oT}}\left(T_{\mathrm{a}}-T_{\mathrm{o}}\right)
$$

Proprielà del ferro - Come è noto (1+1:i) i metalli che presentano gli stati $d$ parzialmente occupati costituiscono il gruppo dei metalli di transizione, mentre i metalli che presentano grli stati $d$ vuoti o totalmente occupati costituiscono il gruppo dei metalli semplici.

Il gruppo dei metalli di transizione ha proprietà più complicate del gruppo dei metalli semplici. Il ferro possiede, nei dieci stati $3 d$. sci elettroni soltanto e quindi appartiene al gruppo di metalli di transizione.

La fase stabile del ferro, a temperatura ordinaria, fase $x$, è costituita da una struttura cubica a corpo centrato, che però diviene instabile tra circa $910^{\circ} \mathrm{C}$ e $1401^{\circ} \mathrm{C}$.

Tra queste due temperature la fase stabile, fase $\beta$, è costituita da una struttura culica a facce centrate. Oltre i $1401^{\circ} \mathrm{C}$ e fino alla temperatura di fusione, la fase stabile, fase $z$, corrisponde di nuovo ad una strutlura cubica a corpo centrato. La fase ó però non è una nuova fase, ma il proseguimento della fase $\alpha$

La temperatura di Debye del ferro, valutata dal calore specifico a hasse temperature e dalle costanti elastiche, ì dell'ordine di $450^{\circ}$. $480^{\prime \prime}$ K. A temperatura ordinaria il valore del calore specifico del ferro ì assai prossimo al valore classico, mentre alla temperatura di fusione supera alquanto tale valore. Questo eccesso del calore specifico ¿ dovuto al contributo degli elettroni degli stati $3 \mathrm{~A}$.

Ammettendo che la fase o rimanga stabile anche sotto pressione, i parametri contenuti nelle equazioni stabilite nel precedente parawrafo potranno essere dedotti dai dati sperimentali ottenuti a temperatura ordinaria. Il fatto poi che il calore specifico al punto di fusione sia un poco superiore al valore classico inciderà soltanto sulla pressione termica, la quale rappresenta soltanto una piccola percen- 
tuale della pressione totale. Quindi come equazione di stato del ferro al punto di fusione potrà essere usata la [5] senza errore troppo sensibile.

Grazie alle misure di compressibilità eseguite da P. W. Bridgmann (") è possibile calcolare il valore di $K_{\mathrm{o}} \mathrm{T}$ e di $\mathrm{I}$.

Mediante i dati di $\mathrm{P}$. W. Bridgmann, che rappresentano l'andamento del volume relativo del ferro, alla temperatura di circa $20^{\prime \prime} \mathrm{C}$, in funzione della pressione, e l'espressione [13] si è ottenuto, col metodo dei minimi quadrati

$$
\begin{gathered}
K_{\mathrm{oT}}=(1,681 \pm 0,003) 10^{\mathrm{i}} \text { bars } \\
2 \Gamma=3,784 \pm 0,193
\end{gathered}
$$

Si possono poi assumere i seguenti dati sperimentali alla temperatura di circa $20^{\circ} \mathrm{C}$ a pressione ordinaria, ossia, sensibilmente, a pressione nulla.

$$
\begin{aligned}
& c_{\mathrm{o}}=7,87 \mathrm{~g} / \mathrm{cm}^{3} \\
& \alpha_{\mathrm{o}}=3,3610^{-\mathrm{b}} \mathrm{gradi} \mathrm{gra}^{-1} \\
& v_{\mathrm{ol}}=6 \mathrm{Km} / \mathrm{sec} \\
& v_{\mathrm{ot}} \geq 3,36 \mathrm{Km} / \mathrm{sec} \\
& \mathrm{T}_{\mathrm{os}}=1808^{\circ} \mathrm{K} \\
& A=55,85
\end{aligned}
$$

Inoltre il ferro possiede un'entropia di fusione pari a 1,97 calorie grado per mole. Da questi dati è facile ricavare il parametro di Grïneisen e i parametri $\gamma_{o l} e \gamma_{o r}$.

Dalla [10] si ha infatti

$$
\dddot{Y}_{\mathrm{o}}=\frac{\alpha_{\mathrm{o}} K_{\mathrm{oT}} A}{3 R}
$$

e quindi

$$
\dddot{\gamma}_{0}=1,610
$$

Quanto a $\gamma_{o l}$ e $\gamma_{o t}$ dalle [9] e dalla [11] si ottiene

$$
\begin{gathered}
\gamma_{\mathrm{ol}}-\frac{3 \gamma_{\mathrm{o}} b+2 \Gamma}{2+3 b} \\
\gamma_{\mathrm{ot}}=\frac{3 \gamma_{\mathrm{o}}(1+b)-\Gamma}{2+3 b}
\end{gathered}
$$


con

le quali forniscono

$$
b=\frac{a^{2}}{1-a^{2}}
$$

$$
\begin{aligned}
& \gamma_{01}=1,750 \\
& \gamma_{01}=1,540
\end{aligned}
$$

La densità for al punto di fusione, calcolata mediante la [14], risulta

$$
\rho_{\text {ous }}=7,43 \quad \mathrm{~g} / \mathrm{rm}^{3}
$$

in buon accordo col valore di 7,3 indicato nell'Handbook of Physical Constans.

Tenendo poi presente che dalle [11] e [12] si ha

$$
\begin{aligned}
& \gamma_{\text {ots }}=\frac{\rho_{0}}{\rho_{0 y}} \gamma_{01} \\
& \gamma_{0 s}=\frac{\rho_{0}}{\rho_{0 y}} \gamma_{0}
\end{aligned}
$$

¿ possibile ottenere i valori di $\gamma_{\text {ots }}$ e $\gamma_{o s}$.

La temperatura di fusione del ferro in funzione della pressione - L'andamento della temperatura di fusione del ferro in funzione della pressione $\dot{e}$ stato calcolato utilizzando $\mathrm{i}$ dati del precedente paragrafo e le relazioni [3] e [5]. La percentuale della pressione termica rispetto alla pressione totale è risultata pari al $15,6 \%$ alla pressione di circa $10^{\prime}$ bars. Con l'aumentare della pressione questa percentuale decresce, riducendosi al $5,6 \%$ alla pressione di circa $+10^{\circ}$ bars.

La variazione della temperatura di fusione con la pressione è stata calcolata mediante la relazione [15]. Purtroppo l'unico confronto possibile con i dati sperimentali può essere effettuato a pressione nulla.

A tale pressione la [15] fornisce un valore di $d T_{\mathrm{s}} / d_{p_{\mathrm{s}}}$ pari a circa $2 ", 97$ per ogni mille bars, in buon accordo col valore di 330 atmosfere per grado, ossia di circa $2^{\prime \prime}, 99$ per ogni mille bars, ottenuto da F. E. Simon $(9)$ con recenti dati sperimentali dalla formula di Clapeyron.

Il massimo della temperatura di fusione del ferro si ha quando $c_{.}=36,4 \mathrm{~g} / \mathrm{cm}^{3}$ ed ha il valore di $8409^{\prime \prime} \mathrm{K}$ circa.

L'andamento della temperatura raggiungibile per compressione adiabatica, a partire dal punto di fusione a pressione nulla, ̀̀ stato ralcolato mediante le relazioni [16] e [17]. 


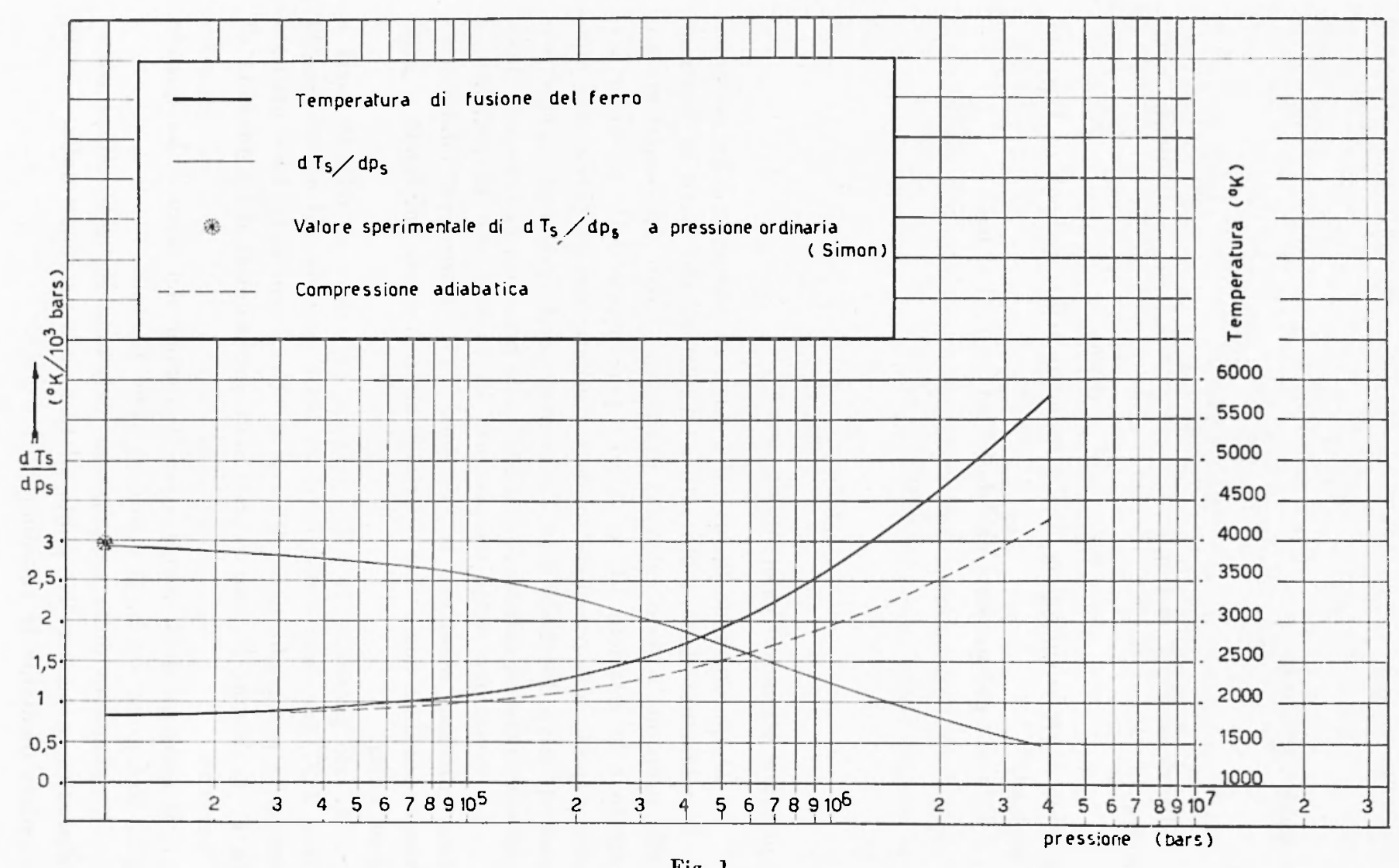

Fig. 1 
Nell'intervallo della pressione che interessa il nucleo della Terra, la compressione adiabatica non mantiene il ferro al punto di fusione.

Il risultato dei calcoli è illustrato nella fiğ. 1 , mentre il confronto con la curva di F. E. Simon viene mostrato nella fig. 2.

Tra le due curve il divario è molto forte e dipende, tra l'altro,

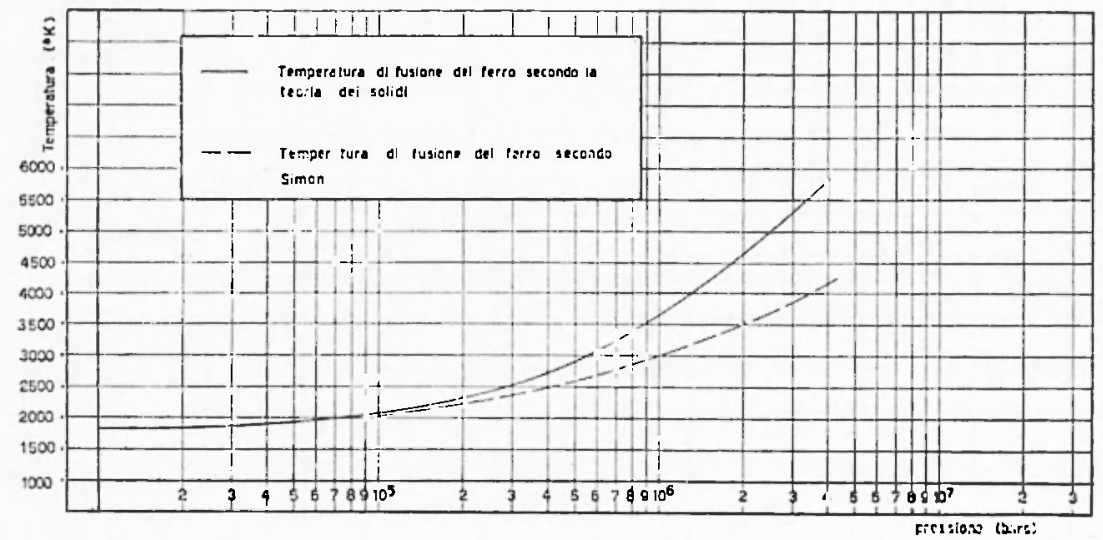

Fig. 2

anche dal fatto che F. E. Simon, per determinare il valore dei parametri che entrano nella sua relazione, ha utilizzato i dati sperimentali dei metalli alcalini.

La curva densitiopressione del forro al punto di fusione. - Dalle relazioni [3] e [5] sono stati anche ottenuti $i$ valori della densiti della faic solida del ferro al punto di fusione, in funzione della pressione.

Nella lig. 3 viene effettuato il confronto con la curva calcolata da H. Jensen mediante il metodo di Thomas e Fermi e con l'isoterma a temperatura ambiente, calcolata da $F$. Birch con la teoria delle deformazioni finte e gli stessi dati sperimentali di P. W. Bridgmann utilizzati in questa ricerca. Dal confronto risulta che la curva di F. Birch i assai prossima alla curva ottenuta dalle relazioni [3] e [5]. La lieve differenza dipende principalmente dalla densiti a pressione nulla, che per la curva di $F$. Birch si riferisce alla temperatura ambiente, mentre per la curva calcolata con le [3] e [5] si riferisce alla temperatura di fusione. Dalla concordanza dellandamento sembra che alle due curve posia attribuirsi un notevole grado di attendibiliti.

Confromto con i dati geofisici - L'andamento della densità nel- 


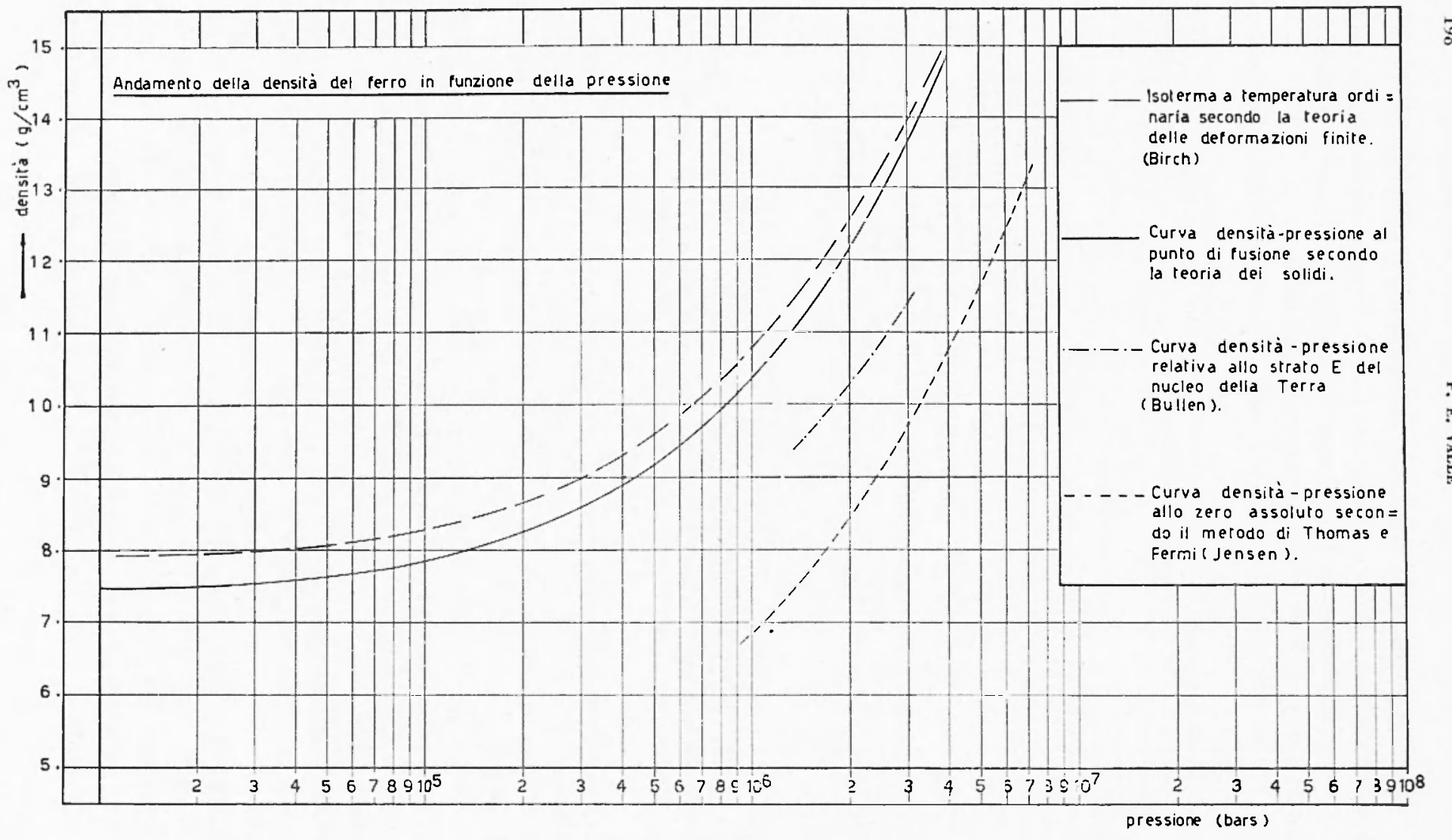

Fig. 3 
l'interno della Terra è stato calcolato da K. E. Bullen $\left.1^{16}\right)$ supponendo l'equilibrio adiabatico e sulla base di due ipotesi estreme. La prima di queste due ipotesi consiste nell'ammettere che la densita vari con continuità attraverso tutto il nucleo della Terra. la seconda che la densita al centro della Terra sia di $10 \mathrm{~g} / \mathrm{cm}^{3}$ maggiore che nel caso precedente.

Nella fig. 3 è riportata la curva densità-pressione relativa allo strato $E$ del nucleo terrestre e corrispondente al caso intermedio $\left({ }^{3}\right)$ Ira le due ipotesi precedenti. Questa curva, a parità di pressione, fornisce in media valori della densita minori di circa il $15 \%$ della densità del ferro al punto di fusione.

L'entitit del divario fra la curva calcolata per il ferro e la curva relativa al nucleo terrestre, tenuto anche conto dei vari fattori d'incertezza che presentano queste curve, mentre non sembra indurre a respingere l'ipotesi secondo la quale il nucleo terrestre sarebbe costituito da un materiale assai simile al ferro meteorico, non ne costituisce neppure una prova definitiva.

Roma -- Istituto Nazionale di Geofisica - Gennaio 1955.

\section{RIASSLNTO}

Mediante la teoria dei solidi viene stimato Iandamento della temperatura e della densita del ferro al punto di fusione, in funzione della pressione.

La temperatura di fusione del ferro cresce con la pressione fino ad un massimo di circa 8409" $K$ che raggiunge quando la densità al

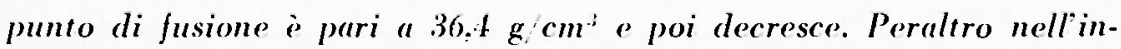
tervallo delle pressioni che si verificano nell'interno della Terra, la compressione adiabatica non mantiene il ferro al punto di fusione.

La curva densiti-pressione del ferro al punto di fusione presenta un andamento assai prossimo allisoterma a temperatura ambionte calcolata da F. Birch con la teoria delle deformazioni fuite.

La curva densità-pressione di K. E. Bullen, relativa allo strato E del nucleo della Terra, fornisce, a parità di pressione, valori della densità minori in media di circa il $15 \%$ rispetto alla curva calcolata. 


\section{$S U M M A R I$}

Using the theory of solids the temperature and density of iron at melting as a function of pressure has been estimated.

The iron has a maximum melting-point of about $8409^{\circ} \mathrm{K}$, which results at density of $36,4 \mathrm{~g} / \mathrm{cm}^{3}$. However in the range of pressure of the Earth's interior the melting of iron cannot be achieved solely by reversible adiabatic compression.

The density - pressure curve of iron at melting is very close to the isothermal curve calculated by F. Birch on the basis of the theory of finite strain.

The Bullen's densities for layer $\mathbf{E}$ of the Eartlis core are about $15 \%$ smaller than the values for iron at melting-point.

\section{BIBLIOGR AFIA}

(1) Ransey W. H., On the nature of the Earth's core. Mon. Not. R. Astr. Soc. $5,+109-426$ (1949).

(2) Bullex K. E., The problem of the Earth's density variation. Bull. Seism. Soc. Am. 30, 235-250 (19:40).

(3) BuLLex K. E., The density cariation of the Earth's central core. Bull. Seism. Soc. Am. 32, 19-29 (19.2).

(4) Jensex H., Das Druck-Dichle Diagramm der Elemente bei hoheren Drucken am Temperaturnallpunkt. Zs. Plysik 111, 373-385 11938 .

(5) Etsasstir W. M., Quantum theoretical densities of solids at exstreme compression. Science, 113, 105-107 (1951).

(5) Feysman R. P., N. Metropolis and E. Telder. Equation of state of elements bused on the generalized Fermi-Thomas theory. Plyys. Rev. 75, 1561-1572 (19.19).

(i) Burch F., Elasticitv and constitution of the Earthis interior. J. Gioph. Res. $57,227.286$ (1952).

(8) Bubswaxy $\mathrm{P}$. W., Linear compression to $30.000 \mathrm{Kg} / \mathrm{cm}^{2}$, including rela. tively incompressibile substances. Proc. Ant. Acad. Arts Science $i 7,187-23.4$ (19.19).

(9) Sinox F. E., The melting of iron at high pressures. Nature, 17i, 746 (1953).

(10) Lindunin F. A., Uber die Berechnung molekulare Eigenfrequenzen, Phys. Zs. $11,609.612$ (1910).

(11) Valle P. E. Un'equazione di stuo per i solidi. Annali di Geofisiea, 6, 183-197 (1953).

(12) Valle. P. E., Sul gradiente adiabatico di temperatura nell interno della Terra. Annali di Geofisica 5, 41-53 (1952).

(13) Valle P. E., Sultomogeneiti e sullo stato di equilibrio del mamello interno della Terra. Annali di Geofisica $7,33-4.4$ (1954).

(14) SeItz F., The modern theory of solids. Me Graw-Hill, New York (1918).

(15) Seitz F. Plysics of metals. Mc Graw-Hill, New York (1943).

(16) Brlben K. E., Introduction to the theory of seismology. Cambridge, University Press (1947). 